Open J. Math. Sci., Vol. 2(2018), No. 1, pp. $266-286$

Website: https://pisrt.org/psr-press/journals/oms/

ISSN: 2523-0212 (Online) 2616-4906 (Print)

http://dx.doi.org/10.30538/oms2018.0034

\title{
ANALYTIC FUNCTIONS OF COMPLEX ORDER DEFINED BY NEW DIFFERENTIAL OPERATOR
}

\author{
ABDUSSALAM EGHBIQ, MASLINA DARUS ${ }^{1}$
}

Abstract. In this paper, we introduce and study the classes $S_{n, \mu}(\gamma, \alpha, \beta$, $\lambda, \nu, \varrho, \mho)$ and $R_{n, \mu}(\gamma, \alpha, \beta, \lambda, \nu, \varrho, \mho)$ of functions $f \in A(n)$ with $(\mu) z\left(D_{\lambda, \nu, \varrho}^{\mho+2}\right.$ $(\alpha, \omega) f(z))^{\prime}+(1-\mu) z\left(D_{\lambda, \nu, \varrho}^{\mho+1}(\alpha, \omega) f(z)\right)^{\prime} \neq 0$, where $\nu>0, \varrho, \omega, \lambda, \alpha, \mu \geq$ $0, \mho \in N_{0}, z \in U$ and $D_{\lambda, \nu, \varrho}^{\mho}(\alpha, \omega) f(z): A(n) \longrightarrow A(n)$, is the linear differential operator, newly defined as

$$
D_{\lambda, \nu, \varrho}^{\mho}(\alpha, \omega) f(z)=z-\sum_{k=n}^{\infty}\left(\frac{\nu+k(\varrho+\lambda) \omega^{\alpha}}{\nu}\right)^{\mho} a_{k+1} z^{k+1} .
$$

Several properties such as coefficient estimates, growth and distortion theorems, extreme points, integral means inequalities and inclusion relation for the functions included in the classes $S_{n, \mu}(\gamma, \alpha, \beta, \lambda, \nu, \varrho, \mho, \omega)$ and $R_{n, \mu}(\gamma, \alpha$, $\beta, \lambda, \nu, \varrho, \mho, \omega)$ are given.

Mathematics Subject Classification: 30C45.

Key words and phrases: analytic functions; differential operator; neighbourhood; complex order; negative coefficients.

\section{Introduction and preliminaries}

Let $H$ be the class of functions analytic in $U=\{z:|z|<1\}$ and let $\mathrm{H}[\mathrm{a}, \mathrm{n}]$ be the subclasses of $H$ consisting of functions of the form $f(z)=a+a_{n} z^{n}+$ $a_{n+1} z^{n+1}+a_{n+2} z^{n+2}+\ldots$. Let $A$ be the subclasses of $H$ consisting of functions of the form $f(z)=z+a_{2} z^{2}+a_{3} z^{3}+\ldots$ or

$$
f(z)=z+\sum_{k=2}^{\infty} a_{k} z^{k} .
$$

Received 24 February 2018. Revised 20 September 2018.

1 Corresponding Author

(C) 2018 Abdussalam Eghbiq, Maslina Darus. This is an open access article distributed under the Creative Commons Attribution License, which permits unrestricted use, distribution, and reproduction in any medium, provided the original work is properly cited. 
Let $A(n)$ denote the class of functions $f(z)$ of the form

$$
\begin{aligned}
& f(z)=z-\sum_{k=n}^{\infty} a_{k+1} z^{k+1}, \\
& a_{k+1} \geq 0, n \in\{1,2,3, \ldots\}
\end{aligned}
$$

which are analytic in the open unit disk $U=\{z:|z|<1\}$.

Next, we define $(n, \delta)$-neighbourhood for the functions belonging to class $A(n)$ and also for identity function.

Definition $1.1((n, \delta)$-neighbourhood). By following the earlier investigations by Goodman [1] and Ruscheweyh [2], for any $f(z) \in A(n)$ and $\delta \geq 0$, we define the $(n, \delta)$-neighbourhood of $f$ by

$$
N_{n, \delta}(f)=\left\{g \in A(n): g(z)=z-\sum_{k=n}^{\infty} b_{k+1} z^{k+1} \quad \text { and } \quad \sum_{k=n}^{\infty}(k+1)\left|a_{k+1}-b_{k+1}\right| \leq \delta\right\} .
$$

In particular for the identity function $e(z)=z$ we have

$$
N_{n, \delta}(e)=\left\{g \in A(n): g(z)=z-\sum_{k=n}^{\infty} b_{k+1} z^{k+1} \quad \text { and } \quad \sum_{k=n}^{\infty}(k+1)\left|b_{k+1}\right| \leq \delta\right\} .
$$

We say that the function $f(z) \in A(n)$ is said to be starlike functions of complex order $\gamma$ or $f(z) \in S_{n}^{*}(\gamma)$ if it satisfies the inequality

$$
\Re\left(1+\frac{1}{\gamma}\left(\frac{z\left(f^{\prime}(z)\right)}{f(z)}-1\right)\right)>0, z \in U, \gamma \in C \backslash\{0\} .
$$

Furthermore, a function $f(z) \in A(n)$ is said to be convex functions of complex order $\gamma$ or $f(z) \in C_{n}^{*}(\gamma)$ if it satisfies the inequality

$$
\Re\left(1+\frac{1}{\gamma}\left(\frac{z\left(f^{\prime \prime}(z)\right)}{f^{\prime}(z)}\right)\right)>0, z \in U, \gamma \in C \backslash\{0\} .
$$

The classes $S_{n}^{*}(\gamma)$ and $C_{n}^{*}(\gamma)$ are essentially from the classes of starlike and convex functions of complex order, which were considered by Nasr and Aouf [3] and Wiatrowsky [4] respectively (Refer also [5]). Let $S_{n}(\gamma, \lambda, \beta)$ denote the subclass of $A(n)$ consisting of functions $f(z)$ which satisfy the following inequality:

$$
\left|\frac{1}{\gamma}\left(\frac{\lambda z^{3} f^{\prime \prime \prime}(z)+(1+2 \lambda) z^{2} f^{\prime \prime}(z)+z f^{\prime}(z)}{\lambda z^{2} f^{\prime \prime}(z)+z f^{\prime}(z)}-1\right)\right|<\beta,
$$

where $z \in U, \gamma \in C \backslash\{0\}, 0 \leq \lambda \leq 1,0<\beta \leq 1$.

Let $R_{n}(\gamma, \lambda, \beta)$ denote the subclass of $A(n)$ consisting of functions $f(z)$ which satisfy the following inequality

$$
\left|\frac{1}{\gamma}\left(\lambda z^{2} f^{\prime \prime \prime}(z)+(1+2 \lambda) z f^{\prime \prime}(z)+f^{\prime}(z)-1\right)\right|<\beta,
$$


where $z \in U, \gamma \in C \backslash\{0\}, 0 \leq \lambda \leq 1,0<\beta \leq 1$.

The class $S_{n}(\gamma, \lambda, \beta)$ was studied by Kamali and Akbulut [6]. Since $A$ is the class of functions $f(z)$ of the form $f(z)=z+\sum_{k=2}^{\infty} a_{k} z^{k}$ which are analytic in the open unit disk $U=\{z:|z|<1\}$.

For a function $f$ in $A$ we define the following differential operator:

$$
\begin{gathered}
D_{\lambda, \nu, \varrho}^{0}(\alpha, \omega) f(z)=f(z), \\
D_{\lambda, \nu, \varrho}^{1}(\alpha, \omega) f(z)=\left(\frac{\nu-(\varrho+\lambda) \omega^{\alpha}}{\nu}\right) f(z)+\left(\frac{(\varrho+\lambda) \omega^{\alpha}}{\nu}\right) z f^{\prime}(z), \\
D_{\lambda, \nu, \varrho}^{2}(\alpha, \omega) f(z)=D\left(D_{\lambda, \nu, \varrho}^{1}(\alpha, \omega) f(z)\right), \\
D_{\lambda, \nu, \varrho}^{m}(\alpha, \omega) f(z)=D\left(D_{\lambda, \nu, \varrho}^{m-1}(\alpha, \omega) f(z)\right) .
\end{gathered}
$$

If $f$ given by (1), then from (7) we define the following differential operator

$$
D_{\lambda, \nu, \varrho}^{m}(\alpha, \omega) f(z)=z+\sum_{k=2}^{\infty}\left(\frac{\nu+(k-1)(\varrho+\lambda) \omega^{\alpha}}{\nu}\right)^{m} a_{k} z^{k}
$$

where $f(z) \in A, \nu>0, \varrho, \omega, \lambda, \alpha \geq 0, m \in N_{0}$.

This operator generalizes certain differential operators such as:

(1) $\nu=1, \varrho=0 \quad$ we get

$$
D_{\lambda, 1,0}^{m}(\alpha, \omega) f(z)=z+\sum_{k=2}^{\infty}\left(1+(k-1) \lambda \omega^{\alpha}\right)^{m} a_{k} z^{k}
$$

of Darus and Faisal (2012) (see [7]);

(2) $\alpha=\omega=\nu=1, \varrho=0$ we get

$$
D_{\lambda, 1,0}^{m}(1,1) f(z)=z+\sum_{k=2}^{\infty}(1+\lambda(k-1))^{m} a_{k} z^{k}
$$

of Al-Oboudi (2004) (see [8]);

(3) $\alpha=\omega=\nu=\lambda=1, \varrho=0$ we get

$$
D_{1,1,0}^{m}(1,1) f(z)=z+\sum_{k=2}^{\infty}(k)^{m} a_{k} z^{k}
$$

of Sălăgean (1983) (see [9]);

(4) $\alpha=\omega=\nu=1, \lambda=2, \varrho=0$, we get

$$
D_{2,1,0}^{m}(1,1) f(z)=z+\sum_{k=2}^{\infty}\left(\frac{k+1}{2}\right)^{m} a_{k} z^{k}
$$

of Uralegaddi and Somanatha (1992) (see [10]). 
By using the same processs, we can write the following equalities for the function $f(z)$ belonging to the class $A(n)$,

$$
\begin{gathered}
D_{\lambda, \nu, \varrho}^{0}(\alpha, \omega) f(z)=f(z) \\
D_{\lambda, \nu, \varrho}^{1}(\alpha, \omega) f(z)=\left(\frac{\nu-(\varrho+\lambda) \omega^{\alpha}}{\nu}\right) f(z)+\left(\frac{(\varrho+\lambda) \omega^{\alpha}}{\nu}\right) z f^{\prime}(z) \\
D_{\lambda, \nu, \varrho}^{2}(\alpha, \omega) f(z)=D\left(D_{\lambda, \nu, \varrho}^{1}(\alpha, \omega) f(z)\right) \\
D_{\lambda, \nu, \varrho}^{\mho}(\alpha, \omega) f(z)=D\left(D_{\lambda, \nu, \varrho}^{\mho-1}(\alpha, \omega) f(z)\right) .
\end{gathered}
$$

If $f$ given by (2), then from (9) we define the following new differential operator:

$$
D_{\lambda, \nu, \varrho}^{\mho}(\alpha, \omega) f(z)=z-\sum_{k=n}^{\infty}\left(\frac{\nu+k(\varrho+\lambda) \omega^{\alpha}}{\nu}\right)^{\mho} a_{k+1} z^{k+1}
$$

where $f \in A(n), \nu>0, \varrho, \omega, \lambda, \alpha \geq 0, \mho \in N_{0}$.

Finally, in the term of the generalized Sălăgean differential operator, let $S_{n, \mu}(\gamma, \alpha$, $\beta, \lambda, \nu, \varrho, \mho)$ denote the subclass of $A(n)$ consisting of the functions $f(z)$ which satisfy the inequality

$$
\left|\frac{1}{\gamma}\left(\frac{(\mu) z\left(D_{\lambda, \nu, \varrho}^{\mho+3}(\alpha, \omega) f(z)\right)^{\prime}+(1-\mu) z\left(D_{\lambda, \nu, \varrho}^{\mho+2}(\alpha, \omega) f(z)\right)^{\prime}}{(\mu) z\left(D_{\lambda, \nu, \varrho}^{\mho+2}(\alpha, \omega) f(z)\right)^{\prime}+(1-\mu) z\left(D_{\lambda, \nu, \varrho}^{\mho+1}(\alpha, \omega) f(z)\right)^{\prime}}-1\right)\right|<\beta,
$$

where $f \in A(n), \gamma \in C \backslash\{0\}, \nu>0, \varrho, \omega, \lambda, \alpha, \mu \geq 0, \mho \in N_{0}, z \in U$. Also, let $R_{n, \mu}(\gamma, \alpha, \beta, \lambda, \nu, \varrho, \mho)$ denote the subclass of $A(n)$ consisting of the functions $f(z)$ which satisfy the inequality

$$
\left|\frac{1}{\gamma}\left(\mu z\left(D_{\lambda, \nu, \varrho}^{\mho+3}(\alpha, \omega) f(z)\right)^{\prime}+(1-\mu) z\left(D_{\lambda, \nu, \varrho}^{\mho+2}(\alpha, \omega) f(z)\right)^{\prime}-1\right)\right|<\beta,
$$

where $f \in A(n), \gamma \in C \backslash\{0\}, \nu>0, \varrho, \omega, \lambda, \alpha, \mu \geq 0, \mho \in N_{0}, z \in U$.

Our main work here is to investigate the $(n, \delta)$-neighborhood of the above said classes i.e. $S_{n, \mu}(\gamma, \alpha, \beta, \lambda, \nu, \varrho, \mho)$ and $R_{n, \mu}(\gamma, \alpha, \beta, \lambda, \nu, \varrho, \mho)$. Similar work has been seen for different subclasses done by other authors (see for example ([11, $12,13])$ and of course many others.

\section{Inclusion relations involving $(n, \delta)$-neighbourhood}

In this section we proved the class relation as well as inclusion relation involving $(n, \delta)$-neighborhood for the subclasses $S_{n, \mu}(\gamma, \alpha, \beta, \lambda, \nu, \varrho, \mho)$ and $R_{n, \mu}(\gamma$, $\alpha, \beta, \lambda, \nu, \varrho, \mho)$ which depends on the following lemmas.

Lemma 2.1. Let the function $f(z) \in A(n)$ be defined by (2), then $f(z)$ is in the class $S_{n, \mu}(\gamma, \alpha, \beta, \lambda, \nu, \varrho, \mho, \omega)$ if and only if 


$$
\begin{aligned}
& \sum_{k=n}^{\infty}\left(\frac{\nu+k(\varrho+\lambda) \omega^{\alpha}}{\nu}\right)^{\mho+1}\left(\frac{\nu+\mu k(\varrho+\lambda) \omega^{\alpha}}{\nu}\right) \\
& \times(k+1)\left(\frac{\beta|\gamma| \nu+k(\varrho+\lambda) \omega^{\alpha}}{\nu}\right) a_{k+1} \leqslant \beta|\gamma|,
\end{aligned}
$$

where $f \in A(n), \nu>0, \varrho, \omega, \lambda, \alpha \geq 0, \mho \in N_{0}, z \in U$.

Proof. Let $f(z) \in S_{n, \mu}(\gamma, \alpha, \beta, \lambda, \nu, \varrho, \mho)$, then from (11) we have

$$
\left|\frac{1}{\gamma}\left(\frac{(\mu) z\left(D_{\lambda}^{\mho+3}(\nu, \alpha, \omega) f(z)\right)^{\prime}+(1-\mu) z\left(D_{\lambda}^{\mho+2}(\nu, \alpha, \omega) f(z)\right)^{\prime}}{(\mu) z\left(D_{\lambda}^{\mho+2}(\nu, \alpha, \omega) f(z)\right)^{\prime}+(1-\mu) z\left(D_{\lambda}^{\mho+1}(\nu, \alpha, \omega) f(z)\right)^{\prime}}-1\right)\right|<\beta,
$$

where $f \in A(n), \nu>0, \varrho, \omega, \lambda, \alpha \geq 0, \mho \in N_{0}$, or

$$
\Re\left(\frac{(\mu) z\left(D_{\lambda}^{\mho+3}(\nu, \alpha, \omega) f(z)\right)^{\prime}+(1-\mu) z\left(D_{\lambda}^{\mho+2}(\nu, \alpha, \omega) f(z)\right)^{\prime}}{(\mu) z\left(D_{\lambda}^{\mho+2}(\nu, \alpha, \omega) f(z)\right)^{\prime}+(1-\mu) z\left(D_{\lambda}^{\mho+1}(\nu, \alpha, \omega) f(z)\right)^{\prime}}-1\right)>-\beta|\gamma|,
$$

where $f \in A(n), \nu>0, \varrho, \omega, \lambda, \alpha \geq 0, \mho \in N_{0}$.

This implies that

$$
\Re\left(\frac{-\sum_{k=n}^{\infty} k(\varrho+\lambda) \omega^{\alpha}\left(\frac{\nu+\mu k(\varrho+\lambda) \omega^{\alpha}}{\nu}\right)(k+1)\left(\frac{\nu+k(\varrho+\lambda) \omega^{\alpha}}{\nu}\right)^{\mho+1} a_{k+1} z^{k+1}}{z-\sum_{k=n}^{\infty}\left(\frac{\nu+\mu k(\varrho+\lambda) \omega^{\alpha}}{\nu}\right)(k+1)\left(\frac{\nu+k(\varrho+\lambda) \omega^{\alpha}}{\nu}\right)^{\mho+1} a_{k+1} z^{k+1}}\right)>-\beta|\gamma|,
$$

where $f \in A(n), \gamma \in C \backslash\{0\}, \nu>0, \varrho, \omega, \lambda, \alpha \geq 0, \mho \in N_{0}, z \in U$, after taking the limit when $z \longrightarrow 1^{-}$and simplifying, we get

$$
\begin{aligned}
& \sum_{k=n}^{\infty}\left(\frac{\nu+k(\varrho+\lambda) \omega^{\alpha}}{\nu}\right)^{\mho+1}\left(\frac{\nu+\mu k(\varrho+\lambda) \omega^{\alpha}}{\nu}\right) \\
& \times(k+1)\left(\frac{\beta|\gamma| \nu+k(\varrho+\lambda) \omega^{\alpha}}{\nu}\right) a_{k+1} \leq \beta|\gamma|,
\end{aligned}
$$

where $\left.f \in A(n), \gamma \in C \backslash\{0\}, \nu>0, \varrho, \omega, \lambda, \alpha \geq 0, \mho \in N_{0}, z \in U\right)$.

Conversely, by applying the hypothesis (13) and letting $|z|=1$ we get

$$
\begin{aligned}
& \left|\frac{(\mu) z\left(D_{\lambda, \nu, \varrho}^{\mho+3}(\alpha, \omega) f(z)\right)^{\prime}+(1-\mu) z\left(D_{\lambda, \nu, \varrho}^{\mho+2}(\alpha, \omega) f(z)\right)^{\prime}}{(\mu) z\left(D_{\lambda, \nu, \varrho}^{\mho+2}(\alpha, \omega) f(z)\right)^{\prime}+(1-\mu) z\left(D_{\lambda, \nu, \varrho}^{\mho+1}(\alpha, \omega) f(z)\right)^{\prime}}-1\right| \\
= & \left|\frac{-\sum_{k=n}^{\infty} k(\varrho+\lambda) \omega^{\alpha}\left(\frac{\nu+\mu k(\varrho+\lambda) \omega^{\alpha}}{\nu}\right)(k+1)\left(\frac{\nu+k(\varrho+\lambda) \omega^{\alpha}}{\nu}\right)^{\mho+1} a_{k+1} z^{k+1}}{z-\sum_{k=n}^{\infty}\left(\frac{\nu+\mu k(\varrho+\lambda) \omega^{\alpha}}{\nu}\right)(k+1)\left(\frac{\nu+k(\varrho+\lambda) \omega^{\alpha}}{\nu}\right)^{\mho+1} a_{k+1} z^{k+1}}\right| \\
\leq & \left|\frac{\beta|\gamma|\left[1-\sum_{k=n}^{\infty}\left(\frac{\nu+\mu k(\varrho+\lambda) \omega^{\alpha}}{\nu}\right)(k+1)\left(\frac{\nu+k(\varrho+\lambda) \omega^{\alpha}}{\nu}\right)^{\mho+1} a_{k+1}\right]}{1-\sum_{k=n}^{\infty}\left(\frac{\nu+\mu k(\varrho+\lambda) \omega^{\alpha}}{\nu}\right)(k+1)\left(\frac{\nu+k(\varrho+\lambda) \omega^{\alpha}}{\nu}\right)^{\mho+1} a_{k+1} z^{k+1}}\right|=\beta|\gamma| .
\end{aligned}
$$


where $f \in A(n), \gamma \in C \backslash\{0\}, \nu>0, \varrho, \omega, \lambda, \alpha \geq 0, \mho \in N_{0}, z \in U$.

This implies that $f(z) \in S_{n, \mu}(\gamma, \alpha, \beta, \lambda, \nu, \varrho, \mho, \omega)$.

Corollary 2.2. Let the function $f$ which is defined by (2) be in the class $S_{n, \mu}(\gamma, \alpha, \beta, \lambda, \nu, \varrho, \mho, \omega)$. Then we have

$$
a_{k+1} \leq \frac{\beta|\gamma|}{\left(\frac{\nu+k(\varrho+\lambda) \omega^{\alpha}}{\nu}\right)^{\mho+1}\left(\frac{\nu+\mu k(\varrho+\lambda) \omega^{\alpha}}{\nu}\right)(k+1)\left(\frac{\beta|\gamma| \nu+k(\varrho+\lambda) \omega^{\alpha}}{\nu}\right)}, k \geq n
$$

where $\nu>0, \varrho, \omega, \lambda, \alpha \geq 0, \mho \in N_{0}$.

Lemma 2.3. Let the function $f(z) \in A(n)$ be defined by (2), then $f(z)$ is in the class $R_{n, \mu}(\gamma, \alpha, \beta, \lambda, \nu, \varrho, \mho, \omega)$ if and only if

$$
\sum_{k=n}^{\infty}\left(\frac{\nu+k(\varrho+\lambda) \omega^{\alpha}}{\nu}\right)^{\mho+2}\left(\frac{2 \nu+\mu k(\varrho+\lambda) \omega^{\alpha}}{\nu}\right)(k+1) a_{k+1} \leqslant \beta|\gamma| .
$$

where $\left(f \in A(n), \nu>0, \varrho, \omega, \lambda, \alpha \geq 0, \mho \in N_{0}, z \in U\right.$.

Proof. Same as Lemma 2.1

Theorem 2.4. Let $f(z) \in A(n)$, then $S_{n, \mu}(\gamma, \alpha, \beta, \lambda, \nu, \varrho, \mho) \subset N_{n, \delta}(e)$ if

$$
\delta=\frac{\beta|\gamma|}{\left(\frac{\nu+n(\varrho+\lambda) \omega^{\alpha}}{\nu}\right)^{\mho+1}\left(\frac{\nu+\mu n(\varrho+\lambda) \omega^{\alpha}}{\nu}\right)(n+1)\left(\frac{\beta|\gamma| \nu+n(\varrho+\lambda) \omega^{\alpha}}{\nu}\right)}
$$

where $\nu>0, \varrho, \omega, \lambda, \alpha \geq 0, \mho \in N_{0}, z \in U$.

Proof. Let $f(z) \in S_{n, \mu}(\gamma, \alpha, \beta, \lambda, \nu, \varrho, \mho)$, then from (13) we get

$$
\begin{aligned}
& \sum_{k=n}^{\infty}\left(\frac{\nu+k(\varrho+\lambda) \omega^{\alpha}}{\nu}\right)^{\mho+1}\left(\frac{\nu+\mu k(\varrho+\lambda) \omega^{\alpha}}{\nu}\right) \\
& \times(k+1)\left(\frac{\beta|\gamma| \nu+k(\varrho+\lambda) \omega^{\alpha}}{\nu}\right) a_{k+1} \leq \beta|\gamma|,
\end{aligned}
$$

where $f \in A(n), \gamma \in C \backslash\{0\}, \nu>0, \varrho, \omega, \lambda, \alpha \geq 0, \mho \in N_{0}, z \in U$. or

$$
\begin{aligned}
& \left(\frac{\nu+n(\varrho+\lambda) \omega^{\alpha}}{\nu}\right)^{\mho+1}\left(\frac{\nu+\mu n(\varrho+\lambda) \omega^{\alpha}}{\nu}\right)(n+1) \\
& \times\left(\frac{\beta|\gamma| \nu+n(\varrho+\lambda) \omega^{\alpha}}{\nu}\right) \sum_{k=n}^{\infty}\left|a_{k+1}\right| \leq \beta|\gamma|,
\end{aligned}
$$

where $f \in A(n), \gamma \in C \backslash\{0\}, \nu>0, \varrho, \omega, \lambda, \alpha \geq 0, \mho \in N_{0}, z \in U$. This implies that 


$$
\begin{aligned}
& \sum_{k=n}^{\infty} a_{k+1} \\
& \leq \frac{\beta|\gamma|}{\left(\frac{\nu+n(\varrho+\lambda) \omega^{\alpha}}{\nu}\right)^{\mho+1}\left(\frac{\nu+\mu n(\varrho+\lambda) \omega^{\alpha}}{\nu}\right)(n+1)\left(\frac{\beta|\gamma| \nu+n(\varrho+\lambda) \omega^{\alpha}}{\nu}\right)}
\end{aligned}
$$

where $f \in A(n), \gamma \in C \backslash\{0\}, \nu>0, \varrho, \omega, \lambda, \alpha \geq 0, \mho \in N_{0}, z \in U$. By using (13) we have

$$
\begin{aligned}
& \sum_{k=n}^{\infty}\left(\frac{\nu+k(\varrho+\lambda) \omega^{\alpha}}{\nu}\right)^{\mho+1}\left(\frac{\nu+\mu k(\varrho+\lambda) \omega^{\alpha}}{\nu}\right) \\
& \times(k+1)\left(\frac{\beta|\gamma| \nu+1-1+k(\varrho+\lambda) \omega^{\alpha}}{\nu}\right) a_{k+1} \leq \beta|\gamma|,
\end{aligned}
$$

where $f \in A(n), \gamma \in C \backslash\{0\}, \nu>0, \varrho, \omega, \lambda, \alpha \geq 0, \mho \in N_{0}$, therefore

$$
\begin{aligned}
& \left(\frac{\nu+n(\varrho+\lambda) \omega^{\alpha}}{\nu}\right)^{\mho+1}\left(\frac{\nu+\mu n(\varrho+\lambda) \omega^{\alpha}}{\nu}\right)\left(\frac{\nu+n(\varrho+\lambda) \omega^{\alpha}}{\nu}\right) \sum_{k=n}^{\infty} a_{k+1} \\
& \leq \beta|\gamma|+(1-\beta|\gamma|)\left(\frac{\nu+n(\varrho+\lambda) \omega^{\alpha}}{\nu}\right)^{\mho+1}\left(\frac{\nu+\mu n(\varrho+\lambda) \omega^{\alpha}}{\nu}\right)(n+1) \sum_{k=n}^{\infty} a_{k+1} \\
& \leq \beta|\gamma|+(1-\beta|\gamma|)\left(\frac{\nu+n(\varrho+\lambda) \omega^{\alpha}}{\nu}\right)^{\mho+1}\left(\frac{\nu+\mu n(\varrho+\lambda) \omega^{\alpha}}{\nu}\right)(n+1) \\
& \times \frac{\beta|\gamma|}{\left(\frac{\nu+n(\varrho+\lambda) \omega^{\alpha}}{\nu}\right)^{\mho+1}\left(\frac{\nu+\mu n(\varrho+\lambda) \omega^{\alpha}}{\nu}\right)(n+1)\left(\frac{\beta|\gamma| \nu+n(\varrho+\lambda) \omega^{\alpha}}{\nu}\right)} \\
& \leq \frac{\beta|\gamma|\left(\frac{\nu+n(\varrho+\lambda) \omega^{\alpha}}{\nu}\right)}{\left(\frac{\nu \beta|\gamma|+n(\varrho+\lambda) \omega^{\alpha}}{\nu}\right)},
\end{aligned}
$$

where $f \in A(n), \nu \neq 0, \varrho, \omega, \lambda, \alpha \geq 0, \mho \in N_{0}, z \in U$. Hence $\sum_{k=n}^{\infty}(k+1) a_{k+1} \leq$ $\frac{\beta|\gamma|}{\left(\frac{\nu+n(\varrho+\lambda) \omega^{\alpha}}{\nu}\right)^{\mho+1}\left(\frac{\nu+\mu n(\varrho+\lambda) \omega^{\alpha}}{\nu}\right)\left(\frac{\beta|\gamma| \nu+n(\varrho+\lambda) \omega^{\alpha}}{\nu}\right)}=\delta$. Hence by using (4), we conclude that $f(z) \in N_{n, \delta}(e)$, this implies that

$$
S_{n, \mu}(\gamma, \alpha, \beta, \lambda, \nu, \varrho, \mho) \in N_{n, \delta}(e)
$$


Using the same technique of the proof of the Theorem 2.4, we proved the following theorem.

Theorem 2.5. Let $f(z) \in A(n)$, then $R_{n, \mu}(\gamma, \alpha, \beta, \lambda, \nu, \varrho, \mho) \subset N_{n, \delta}(e)$ if

$$
\delta=\frac{\beta|\gamma|}{\left(\frac{\nu+n(\varrho+\lambda) \omega^{\alpha}}{\nu}\right)^{\mho+2}\left(\frac{2 \nu+\mu n(\varrho+\lambda) \omega^{\alpha}}{\nu}\right)}
$$

where $f \in A(n), \nu>0, \varrho, \omega, \lambda, \alpha \geq 0, \mho \in N_{0}, z \in U$.

Proof. The proof for this theorem similar to that given above and we omit it.

3. Neighbourhood properties for $\left.S_{n, \mu}^{\tau}(\gamma, \alpha, \beta, \lambda, \nu, \varrho, \mho)\right)$ and

$$
R_{n, \mu}^{\tau}(\gamma, \alpha, \beta, \lambda, \nu, \varrho, \mho)
$$

In this section, we determine the neighbourhood for each of the classes $S_{n, \mu}^{\tau}(\gamma, \alpha, \beta$, $\lambda, \nu, \varrho, \mho)$ and $R_{n, \mu}^{\tau}(\gamma, \alpha, \beta, \lambda, \nu, \varrho, \mho)$. A function $f(z) \in A(n)$ is said to be in the class $S_{n, \mu}^{\tau}(\gamma, \alpha, \beta, \lambda, \nu, \varrho, \mho)$ if there exists a function $g(z) \in S_{n, \mu}(\gamma, \alpha, \beta, \lambda, \nu, \varrho, \mho)$ such that

$$
\left|\frac{f(z)}{g(z)}-1\right|<1-\tau, z \in U, \tau \geq 0 .
$$

Similarly, a function $f(z) \in A(n)$ is said to be in the class $R_{n, \mu}^{\tau}(\gamma, \alpha, \beta, \lambda, \nu, \varrho, \mho)$ if there exists a function $g(z) \in R_{n, \mu}(\gamma, \alpha, \beta, \lambda, \nu, \varrho, \mho)$ satisfying the same inequality

$$
\left|\frac{f(z)}{g(z)}-1\right|<1-\tau, z \in U, \tau \geq 0 .
$$

Theorem 3.1. Let $g(z) \in S_{n, \mu}(\gamma, \alpha, \beta, \lambda, \nu, \varrho, \mho)$ and

$$
\tau=1-\frac{\delta\left(\frac{\nu+n(\varrho+\lambda) \omega^{\alpha}}{\nu}\right)^{\mho+1}\left(\frac{\nu+\mu n(\varrho+\lambda) \omega^{\alpha}}{\nu}\right)\left(\frac{\beta|\gamma| \nu+n(\varrho+\lambda) \omega^{\alpha}}{\nu}\right)}{\left(\frac{\nu+n(\varrho+\lambda) \omega^{\alpha}}{\nu}\right)^{\mho+1}\left(\frac{\nu+\mu n(\varrho+\lambda) \omega^{\alpha}}{\nu}\right)\left(\frac{\beta|\gamma| \nu+n(\varrho+\lambda) \omega^{\alpha}}{\nu}\right)-\beta|\gamma|}
$$

where $f \in A(n), \nu>0, \varrho, \omega, \lambda, \alpha \geq 0, \mho \in N_{0}, z \in U$, then

$$
N_{n, \delta}(g) \subset S_{n, \mu}^{\tau}(\gamma, \alpha, \beta, \lambda, \nu, \varrho, \mho) .
$$

Proof. Let $f \in N_{n, \delta}(g)$, then from (3) we can write that

$$
\sum_{k=n}^{\infty}(k+1)\left|a_{k+1}-b_{k+1}\right| \leq \delta .
$$

This implies that

$$
\sum_{k=n}^{\infty}\left|a_{k+1}-b_{k+1}\right| \leq \frac{\delta}{n+1} .
$$

Since it is given that $g(z) \in S_{n, \mu}(\gamma, \alpha, \beta, \lambda, \nu, \varrho, \mho)$, so from (13) we can write that 


$$
\sum_{k=n}^{\infty} b_{k+1} \leq \frac{\beta|\gamma|}{\left(\frac{\nu+n(\varrho+\lambda) \omega^{\alpha}}{\nu}\right)^{\mho+1}\left(\frac{\nu+\mu n(\varrho+\lambda) \omega^{\alpha}}{\nu}\right)(n+1)\left(\frac{\beta|\gamma| \nu+n(\varrho+\lambda) \omega^{\alpha}}{\nu}\right)}
$$

Now

$$
\begin{aligned}
& \left|\frac{f(z)}{g(z)}-1\right|<\frac{\sum_{k=n}^{\infty}\left|a_{k+1}-b_{k+1}\right|}{1-\sum_{k=n}^{\infty} b_{k+1}} \\
& \leq \frac{\delta}{n+1} \cdot \frac{\left(\frac{\nu+n(\varrho+\lambda) \omega^{\alpha}}{\nu}\right)^{\mho+1}\left(\frac{\nu+\mu n(\varrho+\lambda) \omega^{\alpha}}{\nu}\right)(n+1)\left(\frac{\beta|\gamma| \nu+n(\varrho+\lambda) \omega^{\alpha}}{\nu}\right)}{\left(\frac{\nu+n(\varrho+\lambda) \omega^{\alpha}}{\nu}\right)^{\mho+1}\left(\frac{\nu+\mu n(\varrho+\lambda) \omega^{\alpha}}{\nu}\right)(n+1)\left(\frac{\beta|\gamma| \nu+n(\varrho+\lambda) \omega^{\alpha}}{\nu}\right)-\beta|\gamma|} \\
& =\frac{\delta\left(\frac{\nu+n(\varrho+\lambda) \omega^{\alpha}}{\nu}\right)^{\mho+1}\left(\frac{\nu+\mu n(\varrho+\lambda) \omega^{\alpha}}{\nu}\right)\left(\frac{\beta|\gamma| \nu+n(\varrho+\lambda) \omega^{\alpha}}{\nu}\right)}{\left(\frac{\nu+n(\varrho+\lambda) \omega^{\alpha}}{\nu}\right)^{\mho+1}\left(\frac{\nu+\mu n(\varrho+\lambda) \omega^{\alpha}}{\nu}\right)\left(\frac{\beta|\gamma| \nu+n(\varrho+\lambda) \omega^{\alpha}}{\nu}\right)-\beta|\gamma|} \\
& =1-\tau,
\end{aligned}
$$

this implies that $f \in S_{n, \mu}^{\tau}(\gamma, \alpha, \beta, \lambda, \nu, \varrho, \mho)$, therefore

$$
N_{n, \delta}(g) \subset S_{n, \mu}^{\tau}(\gamma, \alpha, \beta, \lambda, \nu, \varrho, \mho)
$$

Similarly, by using the same technique of Theorem 3.1 we proved the following theorem.

Theorem 3.2. Let $g(z) \in R_{n, \mu}(\gamma, \alpha, \beta, \lambda, \nu, \varrho, \mho)$ and

$$
\tau=1-\frac{\delta\left(\frac{\nu+n(\varrho+\lambda) \omega^{\alpha}}{\nu}\right)^{\mho+2}\left(\frac{2 \nu+\mu n(\varrho+\lambda) \omega^{\alpha}}{\nu}\right)}{\left(\frac{\nu+n(\varrho+\lambda) \omega^{\alpha}}{\nu}\right)^{\mho+2}\left(\frac{2 \nu+\mu n(\varrho+\lambda) \omega^{\alpha}}{\nu}\right)(n+1)-\beta|\gamma|}
$$

where $f(z) \in A(n), \nu>0, \varrho, \omega, \lambda, \alpha \geq 0, \mho \in N_{0}, z \in U$, then

$$
N_{n, \delta}(g) \subset R_{n, \mu}^{\tau}(\gamma, \alpha, \beta, \lambda, \nu, \varrho, \mho)
$$

Our next work is to investigate several new results like growth and distortion theorems, Hadamard product, extreme points, integral means inequalities and inclusion properties for the function included in the classes $S_{n, \mu}(\gamma, \alpha, \beta, \lambda, \nu, \varrho, \mho, \omega)$ and $R_{n, \mu}(\gamma, \alpha, \beta, \lambda, \nu, \varrho, \mho, \omega)$. Similar work has been seen for different subclasses done by other authors (see for example $[14,15,16,17,18,19,20,21]$ ).

\section{Growth and distortion theorems}

A growth and distortion property of function $f$ in the respective classes $S_{n, \mu}(\gamma, \alpha$, $\beta, \lambda, \nu, \varrho, \mho, \omega)$ and $R_{n, \mu}(\gamma, \alpha, \beta, \lambda, \nu, \varrho, \mho, \omega)$ are given as follows: 
Theorem 4.1. If the function $f$ defined by (2) belong to the class $S_{n, \mu}(\gamma, \alpha, \beta, \lambda$, $\nu, \varrho, \mho, \omega)$ and then for $|z|<1$, we have

$$
\begin{aligned}
& |f(z)| \leq|z|+\frac{\left.\beta|\gamma| z\right|^{n+1}}{\left(\frac{\nu+n(\varrho+\lambda) \omega^{\alpha}}{\nu}\right)^{\mho+1}\left(\frac{\nu+\mu n(\varrho+\lambda) \omega^{\alpha}}{\nu}\right)(n+1)\left(\frac{\beta|\gamma| \nu+n(\varrho+\lambda) \omega^{\alpha}}{\nu}\right)} \\
& |f(z)| \geq|z|-\frac{\beta|\gamma||z|^{n+1}}{\left(\frac{\nu+n(\varrho+\lambda) \omega^{\alpha}}{\nu}\right)^{\mho+1}\left(\frac{\nu+\mu n(\varrho+\lambda) \omega^{\alpha}}{\nu}\right)(n+1)\left(\frac{\beta|\gamma| \nu+n(\varrho+\lambda) \omega^{\alpha}}{\nu}\right)}
\end{aligned}
$$

where $f(z) \in A(n), \nu>0, \varrho, \omega, \lambda, \alpha \geq 0, \mho \in N_{0}, z \in U$. The extremal functions are

$$
f(z)=z-\frac{\beta|\gamma|}{\left(\frac{\nu+n(\varrho+\lambda) \omega^{\alpha}}{\nu}\right)^{\mho+1}\left(\frac{\nu+\mu n(\varrho+\lambda) \omega^{\alpha}}{\nu}\right)(n+1)\left(\frac{\beta|\gamma| \nu+n(\varrho+\lambda) \omega^{\alpha}}{\nu}\right)} z^{k+1}, k \geq n .
$$

Proof. Let $f(z) \in S_{n, \mu}(\gamma, \alpha, \beta, \lambda, \nu, \varrho, \mho, \omega)$, then from (13) we get

$$
\begin{aligned}
& \sum_{k=n}^{\infty}\left(\frac{\nu+k(\varrho+\lambda) \omega^{\alpha}}{\nu}\right)^{\mho+1}\left(\frac{\nu+\mu k(\varrho+\lambda) \omega^{\alpha}}{\nu}\right) \\
& \times(k+1)\left(\frac{\beta|\gamma| \nu+k(\varrho+\lambda) \omega^{\alpha}}{\nu}\right)\left|a_{k+1}\right| \leq \beta|\gamma|,
\end{aligned}
$$

where $f \in A(n), \gamma \in C \backslash\{0\}, \nu>0, \varrho, \omega, \lambda, \alpha \geq 0, \mho \in N_{0}, z \in U$, or

$$
\begin{aligned}
& \left(\frac{\nu+n(\varrho+\lambda) \omega^{\alpha}}{\nu}\right)^{\mho+1}\left(\frac{\nu+\mu n(\varrho+\lambda) \omega^{\alpha}}{\nu}\right) \\
& \times(n+1)\left(\frac{\beta|\gamma| \nu+n(\varrho+\lambda) \omega^{\alpha}}{\nu}\right) a_{k+1} \leq \beta|\gamma|,
\end{aligned}
$$

where $f \in A(n), \gamma \in C \backslash\{0\}, \nu>0, \varrho, \omega, \lambda, \alpha \geq 0, \mho \in N_{0}, z \in U$. This implies that

$$
\sum_{k=n}^{\infty} a_{k+1} \leq \frac{\beta|\gamma|}{\left(\frac{\nu+n(\varrho+\lambda) \omega^{\alpha}}{\nu}\right)^{\mho+1}\left(\frac{\nu+\mu n(\varrho+\lambda) \omega^{\alpha}}{\nu}\right)(n+1)\left(\frac{\beta|\gamma| \nu+n(\varrho+\lambda) \omega^{\alpha}}{\nu}\right)}
$$

where $\nu>0, \varrho, \omega, \lambda, \alpha \geq 0, \mho \in N_{0}$.

From (13) we have

$$
|f(z)|=\left|z-\sum_{k=n}^{\infty} a_{k+1} z^{k+1}\right|
$$

or

$$
|f(z)| \geq|z|-\sum_{k=n}^{\infty}\left|a_{k+1}\right|\left|z^{k+1}\right|
$$

This implies that

$$
|f(z)| \geq|z|-\frac{\beta|\gamma|}{\left(\frac{\nu+n(\varrho+\lambda) \omega^{\alpha}}{\nu}\right)^{\mho+1}\left(\frac{\nu+\mu n(\varrho+\lambda) \omega^{\alpha}}{\nu}\right)(n+1)\left(\frac{\beta|\gamma| \nu+n(\varrho+\lambda) \omega^{\alpha}}{\nu}\right)}\left|z^{n+1}\right| .
$$


Similarly

or

$$
|f(z)|=\left|z-\sum_{k=n}^{\infty} a_{k+1} z^{k+1}\right| \leq\left|z+\sum_{k=n}^{\infty} a_{k+1} z^{k+1}\right|,
$$

$$
\begin{aligned}
& |f(z)| \leq|z|+\sum_{k=n}^{\infty}\left|a_{k+1}\right|\left|z^{k+1}\right| \\
& |f(z)| \leq|z|+\frac{\beta|\gamma|}{\left(\frac{\nu+n(\varrho+\lambda) \omega^{\alpha}}{\nu}\right)^{\mho+1}\left(\frac{\nu+\mu n(\varrho+\lambda) \omega^{\alpha}}{\nu}\right)(n+1)\left(\frac{\beta|\gamma| \nu+n(\varrho+\lambda) \omega^{\alpha}}{\nu}\right)}\left|z^{n+1}\right| .
\end{aligned}
$$

Theorem 4.2. If the function $f$ defined by (2) belong to the class $R_{n, \mu}(\gamma, \alpha, \beta, \lambda$, $\nu, \varrho, \mho, \omega)$, then for $|z|<1$, we have

$$
\begin{gathered}
|f(z)| \leq|z|+\frac{\beta|\gamma||z|^{n+1}}{\left(\frac{\nu+n(\varrho+\lambda) \omega^{\alpha}}{\nu}\right)^{\mho+2}\left(\frac{2 \nu+\mu n(\varrho+\lambda) \omega^{\alpha}}{\nu}\right)(n+1)} \\
|f(z)| \geq|z|-\frac{\beta|\gamma||z|^{n+1}}{\left(\frac{\nu+n(\varrho+\lambda) \omega^{\alpha}}{\nu}\right)^{\mho+2}\left(\frac{2 \nu+\mu n(\varrho+\lambda) \omega^{\alpha}}{\nu}\right)(n+1)} \\
\left(f(z) \in A(n), \nu>0, \varrho, \omega, \lambda, \alpha \geq 0, \mho \in N_{0}, z \in U\right) .
\end{gathered}
$$

The extremal functions are

$$
f(z)=z-\frac{\beta|\gamma|}{\left(\frac{\nu+n(\varrho+\lambda) \omega^{\alpha}}{\nu}\right)^{\mho+2}\left(\frac{2 \nu+\mu n(\varrho+\lambda) \omega^{\alpha}}{\nu}\right)(n+1)} z^{k+1}, k \geq n .
$$

Proof. The proof for this theorem is similar to that given above and we omit it.

Theorem 4.3. If the function $f$ defined by (2) belong to the class $S_{n, \mu}(\gamma, \alpha, \beta$, $\lambda, \nu, \varrho, \mho, \omega)$, then for $|z|<1$ we have

$$
\begin{aligned}
& \left|D_{\lambda, \nu, \varrho}^{\mho}(\alpha, \omega) f(z)\right| \\
& \leq|z|+\frac{\beta|\gamma|}{\left(\frac{\nu+\mu n(\varrho+\lambda) \omega^{\alpha}}{\nu}\right)(n+1)\left(\frac{\beta|\gamma| \nu+n(\varrho+\lambda) \omega^{\alpha}}{\nu}\right)}|z|^{n+1} \\
& \left|D_{\lambda, \nu, \varrho}^{\mho}(\alpha, \omega) f(z)\right| \\
& \geq|z|-\frac{\beta|\gamma|}{\left(\frac{\nu+\mu n(\varrho+\lambda) \omega^{\alpha}}{\nu}\right)(n+1)\left(\frac{\beta|\gamma| \nu+n(\varrho+\lambda) \omega^{\alpha}}{\nu}\right)}|z|^{n+1}
\end{aligned}
$$

where $f(z) \in A(n), \nu>0, \varrho, \omega, \lambda, \alpha \geq 0, \mho \in N_{0}, z \in U$. 
Proof. Let $f(z) \in S_{n, \mu}(\gamma, \alpha, \beta, \lambda, \nu, \varrho, \mho, \omega)$, then from (11) we get

$$
\begin{aligned}
& \sum_{k=n}^{\infty}\left(\frac{\nu+k(\varrho+\lambda) \omega^{\alpha}}{\nu}\right)^{\mho+1}\left(\frac{\nu+\mu k(\varrho+\lambda) \omega^{\alpha}}{\nu}\right) \\
& \times(k+1)\left(\frac{\beta|\gamma| \nu+k(\varrho+\lambda) \omega^{\alpha}}{\nu}\right) a_{k+1} \leq \beta|\gamma|,
\end{aligned}
$$

where $f \in A(n), \nu>0, \varrho, \omega, \lambda, \alpha \geq 0, \mho \in N_{0}, z \in U$, or

$$
\begin{aligned}
& \left(\frac{\nu+n(\varrho+\lambda) \omega^{\alpha}}{\nu}\right)^{\mho+1}\left(\frac{\nu+\mu n(\varrho+\lambda) \omega^{\alpha}}{\nu}\right) \\
& \times(n+1)\left(\frac{\beta|\gamma| \nu+n(\varrho+\lambda) \omega^{\alpha}}{\nu}\right) \sum_{k=n}^{\infty}\left|a_{k+1}\right| \leq \beta|\gamma|,
\end{aligned}
$$

where $\nu>0, \varrho, \omega, \lambda, \alpha, \mu \geq 0, \mho \in N_{0}$. This implies that

$$
\begin{aligned}
& \left(\frac{\nu+n(\varrho+\lambda) \omega^{\alpha}}{\nu}\right)^{\mho+1}\left(\frac{\nu+\mu n(\varrho+\lambda) \omega^{\alpha}}{\nu}\right)(n+1)\left(\frac{\beta|\gamma| \nu+n(\varrho+\lambda) \omega^{\alpha}}{\nu}\right) \sum_{k=n}^{\infty}\left|a_{k+1}\right| \\
& \leq \sum_{k=n}^{\infty}\left(\frac{\nu+n(\varrho+\lambda) \omega^{\alpha}}{\nu}\right)^{\mho+1}\left(\frac{\nu+\mu n(\varrho+\lambda) \omega^{\alpha}}{\nu}\right)(n+1)\left(\frac{\beta|\gamma| \nu+n(\varrho+\lambda) \omega^{\alpha}}{\nu}\right)\left|a_{k+1}\right| \\
& \leq \beta|\gamma|
\end{aligned}
$$

or

$$
\begin{aligned}
& \left(\frac{\nu+\mu n(\varrho+\lambda) \omega^{\alpha}}{\nu}\right)(n+1)\left(\frac{\beta|\gamma| \nu+n(\varrho+\lambda) \omega^{\alpha}}{\nu}\right) \\
& \times \sum_{k=n}^{\infty}\left(\frac{\nu+n(\varrho+\lambda) \omega^{\alpha}}{\nu}\right)^{\mho}\left|a_{k+1}\right| \leq \beta|\gamma|
\end{aligned}
$$

where $\nu>0, \varrho, \omega, \lambda, \alpha \geq 0, \mho \in N_{0}$, implies that

$$
\begin{aligned}
& \sum_{k=n}^{\infty}\left(\frac{\nu+n(\varrho+\lambda) \omega^{\alpha}}{\nu}\right)^{\mho}\left|a_{k+1}\right| \\
& \leq \frac{\beta|\gamma|}{\left(\frac{\nu+\mu n(\varrho+\lambda) \omega^{\alpha}}{\nu}\right)(n+1)\left(\frac{\beta|\gamma| \nu+n(\varrho+\lambda) \omega^{\alpha}}{\nu}\right) .}
\end{aligned}
$$

From (10) we have

$$
\begin{gathered}
\left|D_{\lambda, \nu, \varrho}^{\mho}(\alpha, \omega) f(z)\right|=\left|z-\sum_{k=n}^{\infty}\left(\frac{\nu+(k+1-1)(\varrho+\lambda) \omega^{\alpha}}{\nu}\right)^{\mho} a_{k+1} z^{k+1}\right| . \\
\left|D_{\lambda, \nu, \varrho}^{\mho}(\alpha, \omega) f(z)\right| \geq|z|-\sum_{k=n}^{\infty}\left(\frac{\nu+(k+1-1)(\varrho+\lambda) \omega^{\alpha}}{\nu}\right)^{\mho}\left|a_{k+1}\right|\left|z^{k+1}\right| . \\
\quad\left|D_{\lambda, \nu, \varrho}^{\mho}(\alpha, \omega) f(z)\right|
\end{gathered}
$$




$$
\geq|z|-\frac{\beta|\gamma|}{\left(\frac{\nu+\mu n(\varrho+\lambda) \omega^{\alpha}}{\nu}\right)(n+1)\left(\frac{\beta|\gamma| \nu+n(\varrho+\lambda) \omega^{\alpha}}{\nu}\right)}\left|z^{k+1}\right| .
$$

Similarly we can show that

$$
\begin{aligned}
& \left|D_{\lambda, \nu, \varrho}^{\mho}(\alpha, \omega) f(z)\right| \\
& \leq|z|+\frac{\beta|\gamma|}{\left(\frac{\nu+\mu n(\varrho+\lambda) \omega^{\alpha}}{\nu}\right)(n+1)\left(\frac{\beta|\gamma| \nu+n(\varrho+\lambda) \omega^{\alpha}}{\nu}\right)}\left|z^{k+1}\right| .
\end{aligned}
$$

Theorem 4.4. If the function $f$ defined by (2) belong to the class $R_{n, \mu}(\gamma, \alpha, \beta, \lambda$, $\nu, \varrho, \mho, \omega)$, then for $|z|<1$, we have

$$
\begin{aligned}
& \left|D_{\lambda, \nu, \varrho}^{\mho}(\alpha, \omega) f(z)\right| \leq|z|+\left.\frac{\beta|\gamma|}{\left(\frac{2 \nu+\mu n(\varrho+\lambda) \omega^{\alpha}}{\nu}\right)(n+1)} z\right|^{n+1} \mid \\
& \left|D_{\lambda, \nu, \varrho}^{\mho}(\alpha, \omega) f(z)\right| \geq|z|-\left.\frac{\beta|\gamma|}{\left(\frac{2 \nu+\mu n(\varrho+\lambda) \omega^{\alpha}}{\nu}\right)(n+1)} z\right|^{n+1} \mid
\end{aligned}
$$

where $f(z) \in A(n), \nu>0, \varrho, \omega, \lambda, \alpha \geq 0, \mho \in N_{0}, z \in U$.

Theorem 4.5. Let the hypotheses of Theorem 4.1 be satisfied, then

$$
\begin{aligned}
& \left|f^{\prime}(z)\right| \leq 1+\frac{\beta|\gamma||z|^{n}}{\left(\frac{\nu+n(\varrho+\lambda) \omega^{\alpha}}{\nu}\right)^{\mho+1}\left(\frac{\nu+\mu n(\varrho+\lambda) \omega^{\alpha}}{\nu}\right)\left(\frac{\beta|\gamma| \nu+n(\varrho+\lambda) \omega^{\alpha}}{\nu}\right)} \\
& \left|f^{\prime}(z)\right| \geq 1-\frac{\beta|\gamma||z|^{n}}{\left(\frac{\nu+n(\varrho+\lambda) \omega^{\alpha}}{\nu}\right)^{\mho+1}\left(\frac{\nu+\mu n(\varrho+\lambda) \omega^{\alpha}}{\nu}\right)\left(\frac{\beta|\gamma| \nu+n(\varrho+\lambda) \omega^{\alpha}}{\nu}\right)}
\end{aligned}
$$

where $f(z) \in A(n), \nu>0, \varrho, \omega, \lambda, \alpha \geq 0, \mho \in N_{0}, z \in U$.

Theorem 4.6. Let the hypotheses of Theorem 4.2 be satisfied, then

$$
\begin{aligned}
& \left|f^{\prime}(z)\right| \leq 1+\frac{\beta|\gamma||z|^{n}}{\left(\frac{\nu+n(\varrho+\lambda) \omega^{\alpha}}{\nu}\right)^{\mho+2}\left(\frac{2 \nu+\mu n(\varrho+\lambda) \omega^{\alpha}}{\nu}\right)} \\
& \left|f^{\prime}(z)\right| \geq 1-\frac{\beta|\gamma||z|^{n}}{\left(\frac{\nu+n(\varrho+\lambda) \omega^{\alpha}}{\nu}\right)^{\mho+2}\left(\frac{2 \nu+\mu n(\varrho+\lambda) \omega^{\alpha}}{\nu}\right)}
\end{aligned}
$$

where $f(z) \in A(n), \nu>0, \varrho, \omega, \lambda, \alpha \geq 0, \mho \in N_{0}, z \in U$. 
Theorem 4.7. Let the hypotheses of Theorem 4.3 be satisfied, then

$$
\begin{aligned}
& \left|\left(D_{\lambda, \nu, \varrho}^{\mho}(\alpha, \omega) f(z)\right)^{\prime}\right| \leq 1+\frac{\beta|\gamma|}{\left(\frac{\nu+\mu n(\varrho+\lambda) \omega^{\alpha}}{\nu}\right)\left(\frac{\beta|\gamma| \nu+n(\varrho+\lambda) \omega^{\alpha}}{\nu}\right)}|z|^{n} \\
& \left|\left(D_{\lambda, \nu, \varrho}^{\mho}(\alpha, \omega) f(z)\right)^{\prime}\right| \geq 1-\frac{\beta|\gamma|}{\left(\frac{\nu+\mu n(\varrho+\lambda) \omega^{\alpha}}{\nu}\right)\left(\frac{\beta|\gamma| \nu+n(\varrho+\lambda) \omega^{\alpha}}{\nu}\right)}|z|^{n}
\end{aligned}
$$

where $f(z) \in A(n), \nu>0, \varrho, \omega, \lambda, \alpha \geq 0, \mho \in N_{0}, z \in U$.

Theorem 4.8. Let the hypotheses of Theorem 4.4 be satisfied, then

$$
\begin{aligned}
& \left|\left(D_{\lambda, \nu, \varrho}^{\mho}(\alpha, \omega) f(z)\right)^{\prime}\right| \leq 1+\frac{\beta|\gamma|}{\left(\frac{2 \nu+\mu n(\varrho+\lambda) \omega^{\alpha}}{\nu}\right)}|z|^{n} \\
& \left|\left(D_{\lambda, \nu, \varrho}^{\mho}(\alpha, \omega) f(z)\right)^{\prime}\right| \geq 1-\frac{\beta|\gamma|}{\left(\frac{2 \nu+\mu n(\varrho+\lambda) \omega^{\alpha}}{\nu}\right)}|z|^{n}
\end{aligned}
$$

where $f(z) \in A(n), \nu>0, \varrho, \omega, \lambda, \alpha \geq 0, \mho \in N_{0}, z \in U$.

\section{Extreme points}

In this section we discussed extreme points for functions belonging to the classes $S_{n, \mu}(\gamma, \alpha, \beta, \lambda, \nu, \varrho, \mho, \omega)$ and $R_{n, \mu}(\gamma, \alpha, \beta, \lambda, \nu, \varrho, \mho, \omega)$.

Theorem 5.1. (a). Let $f_{1}(z)=z$ and

$$
f_{i}(z)=z-\frac{\beta|\gamma|}{\left(\frac{\nu+k(\varrho+\lambda) \omega^{\alpha}}{\nu}\right)^{\mho+1}\left(\frac{\nu+\mu k(\varrho+\lambda) \omega^{\alpha}}{\nu}\right)(k+1)\left(\frac{\beta|\gamma| \nu+k(\varrho+\lambda) \omega^{\alpha}}{\nu}\right)} z^{i+1}, k \geq n .
$$

then $f \in S_{n, \mu}(\gamma, \alpha, \beta, \lambda, \nu, \varrho, \mho, \omega)$ if and only if it can be expressed in the form $f(z)=\sum_{i=1}^{\infty} \lambda_{i} f_{i}(z)$ where $\lambda_{i} \geq 0$ and $\sum_{i=1}^{\infty} \lambda_{i}=1$.

(b). Let $f_{1}(z)=z$ and

$$
f_{i}(z)=z-\frac{\beta|\gamma|}{\left(\frac{\nu+k(\varrho+\lambda) \omega^{\alpha}}{\nu}\right)^{\mho+2}\left(\frac{2 \nu+\mu k(\varrho+\lambda) \omega^{\alpha}}{\nu}\right)(k+1)} z^{i+1}, k \geq n .
$$

then $f \in R_{n, \mu}(\gamma, \alpha, \beta, \lambda, \nu, \varrho, \mho, \omega)$ if and only if it can be expressed in the form $f(z)=\sum_{i=1}^{\infty} \lambda_{i} f_{i}(z)$ where $\lambda_{i} \geq 0$ and $\sum_{i=1}^{\infty} \lambda_{i}=1$.

Proof. Let $f(z) \in \sum_{i=1}^{\infty} \lambda_{i} f_{i}(z), i=1,2,3, \ldots \lambda_{i} \geq 0$ with $\sum_{i=1}^{\infty} \lambda_{i}=1$. This implies that

$$
f(z) \in \sum_{i=1}^{\infty} \lambda_{i} f_{i}(z)
$$


or

$$
\begin{aligned}
& f(z)=\lambda_{1}(z)+\sum_{i=2}^{\infty} \lambda_{i} \\
& \times\left(z-\frac{\beta|\gamma|}{\left(\frac{\nu+k(\varrho+\lambda) \omega^{\alpha}}{\nu}\right)^{\mho+1}\left(\frac{\nu+\mu k(\varrho+\lambda) \omega^{\alpha}}{\nu}\right)(k+1)\left(\frac{\beta|\gamma| \nu+k(\varrho+\lambda) \omega^{\alpha}}{\nu}\right)} z^{i+1}\right), \\
& \quad f(z)=\lambda_{1}(z)+\sum_{i=2}^{\infty} \lambda_{i}(z)-\sum_{i=2}^{\infty} \lambda_{i} \\
& \times \frac{\beta|\gamma|}{\left(\frac{\nu+k(\varrho+\lambda) \omega^{\alpha}}{\nu}\right)^{\mho+1}\left(\frac{\nu+\mu k(\varrho+\lambda) \omega^{\alpha}}{\nu}\right)(k+1)\left(\frac{\beta|\gamma| \nu+k(\varrho+\lambda) \omega^{\alpha}}{\nu}\right)} z^{i+1}, \\
& \quad f(z)=\sum_{i=1}^{\infty} \lambda_{i}(z)-\sum_{i=2}^{\infty} \lambda_{i} \\
& \quad \times\left(\frac{\beta|\gamma|}{\left(\frac{\nu+k(\varrho+\lambda) \omega^{\alpha}}{\nu}\right)^{\mho+1}\left(\frac{\nu+\mu k(\varrho+\lambda) \omega^{\alpha}}{\nu}\right)(k+1)\left(\frac{\beta|\gamma| \nu+k(\varrho+\lambda) \omega^{\alpha}}{\nu}\right)} z^{i+1}\right), \\
& \quad f(z)=(z)-\sum_{i=2}^{\infty} \lambda_{i} \\
& \quad \times\left(\frac{\beta|\gamma|}{\left(\frac{\nu+k(\varrho+\lambda) \omega^{\alpha}}{\nu}\right)^{\mho+1}\left(\frac{\nu+\mu k(\varrho+\lambda) \omega^{\alpha}}{\nu}\right)(k+1)\left(\frac{\beta|\gamma| \nu+k(\varrho+\lambda) \omega^{\alpha}}{\nu}\right)} z^{i+1}\right) .
\end{aligned}
$$

Since

$$
\begin{gathered}
\sum_{i=2}^{\infty}\left(\frac{\lambda_{i} \beta|\gamma|\left[\left(\frac{\nu+k(\varrho+\lambda) \omega^{\alpha}}{\nu}\right)^{\mho+1}\left(\frac{\nu+\mu k(\varrho+\lambda) \omega^{\alpha}}{\nu}\right)(k+1)\left(\frac{\beta|\gamma| \nu+k(\varrho+\lambda) \omega^{\alpha}}{\nu}\right)\right]}{\left(\frac{\nu+k(\varrho+\lambda) \omega^{\alpha}}{\nu}\right)^{\mho+1}\left(\frac{\nu+\mu k(\varrho+\lambda) \omega^{\alpha}}{\nu}\right)(k+1)\left(\frac{\beta|\gamma| \nu+k(\varrho+\lambda) \omega^{\alpha}}{\nu}\right)} z^{i+1}\right) \\
=\sum_{i=2}^{\infty} \lambda_{i} \beta|\gamma|=\beta|\gamma| \sum_{i=2}^{\infty} \lambda_{i}=\beta|\gamma|\left(1-\lambda_{1}\right)<\beta|\gamma| .
\end{gathered}
$$

The condition (13) for $f(z) \in \sum_{i=1}^{\infty} \lambda_{i} f_{i}(z)$ is satisfied. Thus $f \in S_{n, \mu}(\gamma, \alpha, \beta, \lambda$, $\nu, \varrho, \mho, \omega)$.

Conversely, we suppose that $f \in S_{n, \mu}(\gamma, \alpha, \beta, \lambda, \nu, \varrho, \mho, \omega)$ since

$$
\left|a_{k+1}\right| \leq \frac{\beta|\gamma|}{\left(\frac{\nu+k(\varrho+\lambda) \omega^{\alpha}}{\nu}\right)^{\mho+1}\left(\frac{\nu+\mu k(\varrho+\lambda) \omega^{\alpha}}{\nu}\right)(k+1)\left(\frac{\beta|\gamma| \nu+k(\varrho+\lambda) \omega^{\alpha}}{\nu}\right)}, k \geq n
$$

where $\nu \neq 0, \varrho, \omega, \lambda, \alpha \geq 0, \mho \in N_{0}$. We put

$$
\lambda_{i}=\frac{\left(\frac{\nu+k(\varrho+\lambda) \omega^{\alpha}}{\nu}\right)^{\mho+1}\left(\frac{\nu+\mu k(\varrho+\lambda) \omega^{\alpha}}{\nu}\right)(k+1)\left(\frac{\beta|\gamma| \nu+k(\varrho+\lambda) \omega^{\alpha}}{\nu}\right)}{\beta|\gamma|} a_{i}, k \geq n
$$


where $\nu>0, \varrho, \omega, \lambda, \alpha \geq 0, \mho \in N_{0}$ and $\lambda_{1}=1-\sum_{i=2}^{\infty} \lambda_{i}$, then

$$
f(z)=\sum_{i=1}^{\infty} \lambda_{i} f_{i}(z)
$$

The proof of the second part of Theorem 5.1 is similar to the first part.

\section{Integral means inequalities}

For any two functions $f$ and $g$ analytic in $U, f$ is said to be subordinate to $g$ in $U$ denoted by $f \prec g$ if there exists an analytic function $w$ defined $U$ satisfying $w(0)=0$ and $|w(z)|<1$ such that $f(z)=g(w(z)), z \in U$.

In particular, if the function $g$ is univalent in $U$, the above subordination is equivalent to $f(0)=g(0)$ and $f(U) \subset g(U)$. In 1925, Littlewood [22] proved the following subordination theorem.

Theorem 6.1. If $f$ and $g$ are any two functions, analytic in $U$ with $f \prec g$ then for $\mu>0$ and $z=r e^{i \theta}(0<r<1)$,

$$
\int_{0}^{2 \pi}|f(z)|^{\mu} d \theta \leq \int_{0}^{2 \pi}|g(z)|^{\mu} d \theta .
$$

Theorem 6.2. (a). Let $f \in S_{n, \mu}(\gamma, \alpha, \beta, \lambda, \nu, \varrho, \mho, \omega)$ and $f_{k}$ be defined by

$$
f_{k}(z)=z-\frac{\beta|\gamma|}{\left(\frac{\nu+k(\varrho+\lambda) \omega^{\alpha}}{\nu}\right)^{\mho+1}\left(\frac{\nu+\mu k(\varrho+\lambda) \omega^{\alpha}}{\nu}\right)(k+1)\left(\frac{\beta|\gamma| \nu+k(\varrho+\lambda) \omega^{\alpha}}{\nu}\right)} z^{k+1}, k \geq n .
$$

if there exists an analytic function $w(z)$ given by

$$
[w(z)]^{k}=\frac{\left(\frac{\nu+k(\varrho+\lambda) \omega^{\alpha}}{\nu}\right)^{\mho+1}\left(\frac{\nu+\mu k(\varrho+\lambda) \omega^{\alpha}}{\nu}\right)(k+1)\left(\frac{\beta|\gamma| \nu+k(\varrho+\lambda) \omega^{\alpha}}{\nu}\right)}{\beta|\gamma|} \sum_{k=n}^{\infty} a_{k+1} z^{k},
$$

then for $z=r e^{i \theta}(0<r<1)$,

$$
\int_{0}^{2 \pi}\left|f\left(r e^{i \theta}\right)\right|^{\mu} d \theta \leq \int_{0}^{2 \pi}\left|f_{k}\left(r e^{i \theta}\right)\right|^{\mu} d \theta
$$

(b). Let $f \in R_{n, \mu}(\gamma, \alpha, \beta, \lambda, \nu, \varrho, \mho, \omega)$ and $f_{k}$ be defined by

$$
f_{k}(z)=z-\frac{\beta|\gamma|}{\left(\frac{\nu+k(\varrho+\lambda) \omega^{\alpha}}{\nu}\right)^{\mho+2}\left(\frac{2 \nu+\mu k(\varrho+\lambda) \omega^{\alpha}}{\nu}\right)(k+1)} z^{k+1}, k \geq n .
$$

If there exists an analytic function $w(z)$ given by

$$
[w(z)]^{k}=\frac{\left(\frac{\nu+k(\varrho+\lambda) \omega^{\alpha}}{\nu}\right)^{\mho+2}\left(\frac{2 \nu+\mu k(\varrho+\lambda) \omega^{\alpha}}{\nu}\right)(k+1)}{\beta|\gamma|} \sum_{k=n}^{\infty} a_{k+1} z^{k},
$$


then for $z=r e^{i \theta}(0<r<1)$,

$$
\int_{0}^{2 \pi}\left|f\left(r e^{i \theta}\right)\right|^{\mu} d \theta \leq \int_{0}^{2 \pi}\left|f_{k}\left(r e^{i \theta}\right)\right|^{\mu} d \theta
$$

Proof. (a) We have to show that

$$
\int_{0}^{2 \pi}\left|f\left(r e^{i \theta}\right)\right|^{\mu} d \theta \leq \int_{0}^{2 \pi}\left|f_{k}\left(r e^{i \theta}\right)\right|^{\mu} d \theta
$$

or $\left.\int_{0}^{2 \pi} \mid z-\sum_{k=n}^{\infty} a_{k+1} z^{k+1}\right)\left.\right|^{\mu} d \theta$

$$
\begin{aligned}
& \leq \int_{0}^{2 \pi}\left|z-\frac{\beta|\gamma|}{\left(\frac{\nu+k(\varrho+\lambda) \omega^{\alpha}}{\nu}\right)^{\mho+1}\left(\frac{\nu+\mu k(\varrho+\lambda) \omega^{\alpha}}{\nu}\right)(k+1)\left(\frac{\beta|\gamma| \nu+k(\varrho+\lambda) \omega^{\alpha}}{\nu}\right)} z^{k+1}\right|^{\mu} d \theta \\
& \text { or } \\
& \left.\quad \int_{0}^{2 \pi} \mid 1-\sum_{k=n}^{\infty} a_{k+1} z^{k}\right)\left.\right|^{\mu} d \theta \\
& \quad \leq \int_{0}^{2 \pi}\left|1-\frac{\beta|\gamma|}{\left(\frac{\nu+k(\varrho+\lambda) \omega^{\alpha}}{\nu}\right)^{\mho+1}\left(\frac{\nu+\mu k(\varrho+\lambda) \omega^{\alpha}}{\nu}\right)(k+1)\left(\frac{\beta|\gamma| \nu+k(\varrho+\lambda) \omega^{\alpha}}{\nu}\right)} z^{k}\right|^{\mu} d \theta
\end{aligned}
$$

By using Theorem 6.1 it is enough to show that

$$
\begin{aligned}
& 1-\sum_{k=n}^{\infty} a_{k+1} z^{k} \\
& <1-\frac{\beta|\gamma|}{\left(\frac{\nu+k(\varrho+\lambda) \omega^{\alpha}}{\nu}\right)^{\mho+1}\left(\frac{\nu+\mu k(\varrho+\lambda) \omega^{\alpha}}{\nu}\right)(k+1)\left(\frac{\beta|\gamma| \nu+k(\varrho+\lambda) \omega^{\alpha}}{\nu}\right)} z^{k} .
\end{aligned}
$$

Now by taking

$$
\begin{aligned}
1 & -\sum_{k=n}^{\infty} a_{k+1} z^{k} \\
& =1-\frac{\beta|\gamma|}{\left(\frac{\nu+k(\varrho+\lambda) \omega^{\alpha}}{\nu}\right)^{\mho S+1}\left(\frac{\nu+\mu k(\varrho+\lambda) \omega^{\alpha}}{\nu}\right)(k+1)\left(\frac{\beta|\gamma| \nu+k(\varrho+\lambda) \omega^{\alpha}}{\nu}\right)}(w(z))^{k}
\end{aligned}
$$

and after simplification we get

$$
[w(z)]^{k}=\frac{\left(\frac{\nu+k(\varrho+\lambda) \omega^{\alpha}}{\nu}\right)^{\mho+1}\left(\frac{\nu+\mu k(\varrho+\lambda) \omega^{\alpha}}{\nu}\right)(k+1)\left(\frac{\beta|\gamma| \nu+k(\varrho+\lambda) \omega^{\alpha}}{\nu}\right)}{\beta|\gamma|} \sum_{k=n}^{\infty} a_{k+1} z^{k} .
$$

This implies that $w(0)=0$ and

$$
\left|[w(z)]^{k}\right|=\left|\frac{\left(\frac{\nu+k(\varrho+\lambda) \omega^{\alpha}}{\nu}\right)^{\mho+1}\left(\frac{\nu+\mu k(\varrho+\lambda) \omega^{\alpha}}{\nu}\right)(k+1)\left(\frac{\beta|\gamma| \nu+k(\varrho+\lambda) \omega^{\alpha}}{\nu}\right)}{\beta|\gamma|} \sum_{k=n}^{\infty} a_{k+1} z^{k}\right|,
$$

or

$$
\left|[w(z)]^{k}\right|=\frac{\left(\frac{\nu+k(\varrho+\lambda) \omega^{\alpha}}{\nu}\right)^{\mho+1}\left(\frac{\nu+\mu k(\varrho+\lambda) \omega^{\alpha}}{\nu}\right)(k+1)\left(\frac{\beta|\gamma| \nu+k(\varrho+\lambda) \omega^{\alpha}}{\nu}\right)}{\beta|\gamma|} \sum_{k=n}^{\infty}\left|a_{k+1}\right|\left|z^{k}\right| .
$$

By using (13) we get

$$
\left|[w(z)]^{k}\right| \leq|z|<1
$$


The proof of the second part of the Theorem 6.2 is similar to the first part.

\section{Inclusion properties}

Here we discussed the inclusion properties of the subclasses of analytic functions of complex order denoted by $S_{n, \mu}(\gamma, \alpha, \beta, \lambda, \nu, \varrho, \mho, \omega)$ and $R_{n, \mu}(\gamma, \alpha, \beta, \lambda, \nu, \varrho, \mho$, $\omega)$.

Theorem 7.1. (a) Let $0 \leq \alpha_{1} \leq \alpha_{2} \leq 1,0 \leq \beta_{1} \leq \beta_{2} \leq 1,0 \leq \lambda_{1} \leq \lambda_{2} \leq 1$ and $0 \leq \omega_{1} \leq \omega_{2} \leq 1$. Let a function $f$ be in the class $S_{n, \mu}(\gamma, \alpha, \beta, \lambda, \nu, \varrho, \mho, \omega)$ satisfying

$$
\sum_{k=n}^{\infty}\left(\frac{\nu+k(\varrho+\lambda) \omega^{\alpha}}{\nu}\right)^{\mho+1}\left(\frac{\nu+\mu k(\varrho+\lambda) \omega^{\alpha}}{\nu}\right)(k+1)\left(\frac{\beta|\gamma| \nu+k(\varrho+\lambda) \omega^{\alpha}}{\nu}\right) a_{k+1} \leq \beta|\gamma|
$$

then

- $S_{n, \mu}\left(\gamma, \alpha_{1}, \beta, \lambda, \nu, \varrho, \mho, \omega\right) \subseteq S_{n, \mu}\left(\gamma, \alpha_{2}, \beta, \lambda, \nu, \varrho, \mho, \omega\right)$.

- $S_{n, \mu}\left(\gamma, \alpha, \beta, \lambda_{2}, \nu, \varrho, \mho, \omega\right) \subseteq S_{n, \mu}\left(\gamma, \alpha, \beta, \lambda_{1}, \nu, \varrho, \mho, \omega\right)$.

- $S_{n, \mu}\left(\gamma, \alpha, \beta, \lambda, \nu, \varrho, \mho, \omega_{2}\right) \subseteq S_{n, \mu}\left(\gamma, \alpha, \beta, \lambda, \nu, \varrho, \mho, \omega_{1}\right)$.

- $S_{n, \mu_{2}}(\gamma, \alpha, \beta, \lambda, \nu, \varrho, \mho, \omega) \subseteq S_{n, \mu_{1}}(\gamma, \alpha, \beta, \lambda, \nu, \varrho, \mho, \omega)$.

(b) Let $0 \leq \alpha_{1} \leq \alpha_{2} \leq 1,0 \leq \beta_{1} \leq \beta_{2} \leq 1,0 \leq \lambda_{1} \leq \lambda_{2} \leq 1$ and $0 \leq \omega_{1} \leq \omega_{2} \leq$

1. Let a function $f$ be in the class $R_{n, \mu}(\gamma, \alpha, \beta, \lambda, \nu, \varrho, \mho, \omega)$ satisfying

$$
\sum_{k=n}^{\infty}\left(\frac{\nu+k(\varrho+\lambda) \omega^{\alpha}}{\nu}\right)^{\mho+2}\left(\frac{2 \nu+\mu k(\varrho+\lambda) \omega^{\alpha}}{\nu}\right)(k+1) a_{k+1} \leq \beta|\gamma|
$$

then

- $R_{n, \mu}\left(\gamma, \alpha_{1}, \beta, \lambda, \nu, \varrho, \mho, \omega\right) \subseteq R_{n, \mu}\left(\gamma, \alpha_{2}, \beta, \lambda, \nu, \varrho, \mho, \omega\right)$.

- $R_{n, \mu}\left(\gamma, \alpha, \beta, \lambda_{2}, \nu, \varrho, \mho, \omega\right) \subseteq R_{n, \mu}\left(\gamma, \alpha, \beta, \lambda_{1}, \nu, \varrho, \mho, \omega\right)$.

- $R_{n, \mu}\left(\gamma, \alpha, \beta, \lambda, \nu, \varrho, \mho, \omega_{2}\right) \subseteq R_{n, \mu}\left(\gamma, \alpha, \beta, \lambda, \nu, \varrho, \mho, \omega_{1}\right)$.

- $R_{n, \mu_{2}}(\gamma, \alpha, \beta, \lambda, \nu, \varrho, \mho, \omega) \subseteq R_{n, \mu_{1}}(\gamma, \alpha, \beta, \lambda, \nu, \varrho, \mho, \omega)$.

Proof. (a) To prove $S_{n, \mu}\left(\gamma, \alpha_{1}, \beta, \lambda, \nu, \varrho, \mho, \omega\right) \subseteq S_{n, \mu}\left(\gamma, \alpha_{2}, \beta, \lambda, \nu, \varrho, \mho, \omega\right)$. Since it is given that $0 \leq \alpha_{1} \leq \alpha_{2} \leq 1$, implies

$$
\begin{aligned}
& \sum_{k=n}^{\infty}\left(\frac{\nu+k(\varrho+\lambda) \omega^{\alpha_{2}}}{\nu}\right)^{\mho+1}\left(\frac{\nu+\mu k(\varrho+\lambda) \omega^{\alpha_{2}}}{\nu}\right)(k+1)\left(\frac{\beta|\gamma| \nu+k(\varrho+\lambda) \omega^{\alpha_{2}}}{\nu}\right) a_{k+1} \\
\leq & \sum_{k=n}^{\infty}\left(\frac{\nu+k(\varrho+\lambda) \omega^{\alpha_{1}}}{\nu}\right)^{\mho+1}\left(\frac{\nu+\mu k(\varrho+\lambda) \omega^{\alpha_{1}}}{\nu}\right)(k+1)\left(\frac{\beta|\gamma| \nu+k(\varrho+\lambda) \omega^{\alpha_{1}}}{\nu}\right) a_{k+1}
\end{aligned}
$$


therefore if $f(z) \in S_{n, \mu}\left(\gamma, \alpha_{1}, \beta, \lambda, \nu, \varrho, \mho, \omega\right)$ implies $f(z) \in S_{n, \mu}\left(\gamma, \alpha_{2}, \beta, \lambda, \nu, \varrho\right.$, $\mho, \omega)$. This show that $S_{n, \mu}\left(\gamma, \alpha_{1}, \beta, \lambda, \nu, \varrho, \mho, \omega\right) \subseteq S_{n, \mu}\left(\gamma, \alpha_{2}, \beta, \lambda, \nu, \varrho, \mho, \omega\right)$.

Similarly, to prove that $S_{n, \mu}\left(\gamma, \alpha, \beta, \lambda_{2}, \nu, \varrho, \mho, \omega\right) \subseteq S_{n, \mu}\left(\gamma, \alpha, \beta, \lambda_{1}, \nu, \varrho, \mho, \omega\right)$. Since it is given that $0 \leq \lambda_{1} \leq \lambda_{2} \leq 1$ thus

$$
\begin{aligned}
& \sum_{k=n}^{\infty}\left(\frac{\nu+k\left(\varrho+\lambda_{1}\right) \omega^{\alpha}}{\nu}\right)^{\mho+1}\left(\frac{\nu+\mu k\left(\varrho+\lambda_{1}\right) \omega^{\alpha}}{\nu}\right)(k+1)\left(\frac{\beta|\gamma| \nu+k\left(\varrho+\lambda_{1}\right) \omega^{\alpha}}{\nu}\right) a_{k+1} \\
\leq & \sum_{k=n}^{\infty}\left(\frac{\nu+k\left(\varrho+\lambda_{2}\right) \omega^{\alpha}}{\nu}\right)^{\mho+1}\left(\frac{\nu+\mu k\left(\varrho+\lambda_{2}\right) \omega^{\alpha}}{\nu}\right)(k+1)\left(\frac{\beta|\gamma| \nu+k\left(\varrho+\lambda_{2}\right) \omega^{\alpha}}{\nu}\right) a_{k+1}
\end{aligned}
$$

Therefore, if $f(z) \in S_{n, \mu}\left(\gamma, \alpha, \beta, \lambda_{2}, \nu, \varrho, \mho, \omega\right)$ implies $f(z) \in S_{n, \mu}\left(\gamma, \alpha, \beta, \lambda_{1}, \nu\right.$, $\varrho, \mho, \omega)$. This show that $S_{n, \mu}\left(\gamma, \alpha, \beta, \lambda_{2}, \nu, \varrho, \mho, \omega\right) \subseteq S_{n, \mu}\left(\gamma, \alpha, \beta, \lambda_{1}, \nu, \varrho, \mho, \omega\right)$.

The proof of the remaining parts of the theorem is similar.

\section{Hadamard Product}

Theorem 8.1. Let $f, g \in A(n)$ where $f(z)$ is given in (2) and $g(z)=z-$ $\sum_{k=n}^{\infty} b_{k+1} z^{k+1}$, then the modified Hadamard product $f * g$ is defined by $(f *$ $g)(z)=z-\sum_{k=n}^{\infty} a_{k+1} b_{k+1} z^{k+1}$. (a) If $f(z)=z-\sum_{k=n}^{\infty} a_{k+1} z^{k+1} \in S_{n, \mu}(\gamma, \alpha, \beta$, $\lambda, \nu, \varrho, \mho, \omega)$ and $g(z)=z-\sum_{k=n}^{\infty} b_{k+1} z^{k+1} \in S_{n, \mu}(\gamma, \alpha, \beta, \lambda, \nu, \varrho, \mho, \omega)$ then $(f * g)(z) \in S_{n, \mu}(\gamma, \alpha, \beta, \lambda, \nu, \varrho, \mho, \omega)$.

(b) If $f(z)=z-\sum_{k=n}^{\infty} a_{k+1} z^{k+1} \in R_{n, \mu}(\gamma, \alpha, \beta, \lambda, \nu, \varrho, \mho, \omega)$ and $g(z)=z-$ $\sum_{k=n}^{\infty} b_{k+1} z^{k+1} \in R_{n, \mu}(\gamma, \alpha, \beta, \lambda, \nu, \varrho, \mho, \omega)$, then $(f * g)(z) \in R_{n, \mu}(\gamma, \alpha, \beta, \lambda, \nu, \varrho$, $\mho, \omega)$.

Proof. (a) Since it is given that $f(z)=z-\sum_{k=n}^{\infty} a_{k+1} z^{k+1} \in S_{n, \mu}(\gamma, \alpha, \beta, \lambda, \nu, \varrho$, $\mho, \omega)$, implies

$$
\sum_{k=n}^{\infty}\left(\frac{\nu+k(\varrho+\lambda) \omega^{\alpha}}{\nu}\right)^{\mho+1}\left(\frac{\nu+\mu k(\varrho+\lambda) \omega^{\alpha}}{\nu}\right)(k+1)\left(\frac{\beta|\gamma| \nu+k(\varrho+\lambda) \omega^{\alpha}}{\nu}\right) a_{k+1} \leq \beta|\gamma| .
$$

Similarly $g(z)=z-\sum_{k=n}^{\infty} b_{k+1} z^{k+1} \in R_{n, \mu}(\gamma, \alpha, \beta, \lambda, \nu, \varrho, \mho, \omega)$, implies

$$
\sum_{k=n}^{\infty}\left(\frac{\nu+k(\varrho+\lambda) \omega^{\alpha}}{\nu}\right)^{\mho+1}\left(\frac{\nu+\mu k(\varrho+\lambda) \omega^{\alpha}}{\nu}\right)(k+1)\left(\frac{\beta|\gamma| \nu+k(\varrho+\lambda) \omega^{\alpha}}{\nu}\right) b_{k+1} \leq \beta|\gamma|,
$$

because

$$
\begin{gathered}
\sum_{k=n}^{\infty}\left(\frac{\nu+k(\varrho+\lambda) \omega^{\alpha}}{\nu}\right)^{\mho+1}\left(\frac{\nu+\mu k(\varrho+\lambda) \omega^{\alpha}}{\nu}\right)(k+1)\left(\frac{\beta|\gamma| \nu+k(\varrho+\lambda) \omega^{\alpha}}{\nu}\right) b_{k+1} \\
\leq \sum_{k=n}^{\infty}\left(\frac{\nu+k(\varrho+\lambda) \omega^{\alpha}}{\nu}\right)^{\mho+1}\left(\frac{\nu+\mu k(\varrho+\lambda) \omega^{\alpha}}{\nu}\right)(k+1)\left(\frac{\beta|\gamma| \nu+k(\varrho+\lambda) \omega^{\alpha}}{\nu}\right) a_{k+1} \leq \beta|\gamma| .
\end{gathered}
$$

Other work regarding differential operators for various problems can be found in $[6,7,23,24,25,26,27,28,29]$.

\section{Acknowledgment}

The work here is supported by GUP-2017-064. 


\section{Competing Interests}

The author(s) do not have any competing interests in the manuscript.

\section{REFERENCES}

1. Goodman, A. W. (1957). Univalent functions and nonanalytic curves. Proceedings of the American Mathematical Society, 8, 598-601. https://doi.org/10.1090/S0002-9939-19570086879-9

2. Ruscheweyh, S. (1981). Neighbourhoods of univalent functions. Proc. Amer. Math. Soc., 81, 521-527. https://doi.org/10.1090/S0002-9939-1981-0601721-6

3. Nasr, M. A., \& Aouf, M. K. (1985). Starlike function of complex order. J. Natur. Sci. Math. and Stat., 25, 1-12.

4. Wiatrowski, P. (1970). On the coefficients of a some family of holomorphic functions. Zeszyty Nauk, Uniw. Ldz. Nauk. Mat-Przyrod., 2(39), 75-85.

5. Duren, P. L. (1983). Univalent functions in: A series of comprehensive studies in Mathematics. Springer-Verlag, New York, Berlin, Heidelberg, Tokyo.

6. Kamali, M., \& Akbulut, S. (2002). On a subclass of certain convex functions with negative coefficients. J. Math. Comput., 145, 341-350. http://dx.doi.org/10.1016/S00963003(02)00491-5

7. Darus, M., \& Faisal, I. (2012). Some subclasses of analytic functions of complex order defined by new differential operator. Tamkang Journal of Mathematics, 43, 223-242. http://dx.doi.org/10.5556/j.tkjm.43.2012.740

8. Al-Oboudi, 'F. M. (2004). On univalent functions defined by a generalized Salagean operator. Int.J. Math. Math. Sci.,2004(27), 1429-1436. http://dx.doi.org/10.1155/S0161171204108090

9. Salagean, G. S. (1983). Subclasses of univalent Functions. Lecture Notes in Mathematics 1013, Spriger-Verlag, 362-372. https://doi.org/10.1007/BFb0066543

10. Uralegaddi, B. A., \& Somanatha, C. (1992). Certain classes of univalent functions. In: Current Topics in Analytic Function Theory. Eds. H.M. Srivastava and S. Owa. World Scientific Publishing Company, Singapore, 371-374. https://doi.org/10.1142/9789814355896_0032

11. Altintas, O., \& Owa, S. (1996). Neighbourhoods of certain analytic functions with negative coefficents. Internat. J. Math. Math. Sci., 19, 797-800. https://doi.org/10.1016/S08939659(99)00187-1

12. Altintas, O., Ozkan, S., \& Srivastava, H. M. (2000). Neighbourhoods of a class of analytic functions with negative coefficients. Appl. Math. Lett. , 13(3), 63-67. https://doi.org/10.1016/S0893-9659(99)00187-1

13. Altintas, O., Ozkan, S., \& Srivastava, H. M. (2004). Neighborhoods of a certain family of multivalent functions with negative coefficients. Comput. Math. Appl., 1(10), 1667-1672. https://doi.org/10.1016/j.camwa.2004.06.014

14. Joshi, S.S. (2008). A certain class of analytic functions associated with fractional derivative operators. Tamsui Oxford Journal of Mathematical Sciences, 24, 201-214.

15. Al Attiya, A. A., \& Aouf, M. K. (2007). A study on certain class of analytic functions defined by Ruscheweyh derivative. Soochow Journal of Mathematics, 33, 273-289.

16. Raducanu, D., \& Orhan, H. (2010). Subclasses of analytic Functions defined by a generalized differential operator. Int. Jour. Math. Anal., 4, 1-15.

17. Mahzoon, H. \& Latha, S. (2007). Certain subclass of analytic functions with negative coffecient defined by a generalized Salagean operator. General Mathematics, 15, 69-82.

18. Mahzoon, H. \& Latha, S. (2010). On the classes of analytic functions involving Al-Oboudi operator. Int. Jour. Math. Anal., 4, 193-199.

19. Aouf, M.K., \& Darwish, H. E. (1994). On new class of analytic functions with negative coefficients. II. Bull. Korean Math. Sci., 31, 269-287. 
20. Al-Refai, O., \& Darus, M. (2009). On new bijective convolution operator acting for analytic functions. Journal of Mathematics and statistics, 5(1), 77-87. https://doi.org/10.3844/jmssp.2009.77.87

21. Halim, S. A., Janteng, A., \& Darus, M. (2007). Classes with negative coefficients and starlike with respect to other points. International Mathematical Forum, 2(46), 2261-2268.

22. Littlewood, J. E. (1925). On inequalities in the theory of functions. Proc. Lond. Math. Soc., 23, 481-519. https://doi.org/10.1112/plms/s2-23.1.481

23. Ghanim, F. \& Darus, M. (2008). On certain class of analytic function with fixed second positive coefficient. Int. Jour. Math. Anal., 2, 55-66.

24. Al-Shaqsi, K. \& Darus, M. (2008). An operator defined by convolution involving the polylogarithmics functions. Journal of Mathematics and Statistics, 4(1), 46-50. https://doi.org/10.3844/jmssp.2008.46.50

25. Darus, M., \& Ibrahim, R. W. (2009). New classes containing generalization of differential operator. Appl. Math. Sci., 3, 2507-2515.

26. Al-Abbadi, M. H., \& Darus, M. (2010). Differential subordination defined by generalised derivative operator for analytic functions. Inter. Jour. Math. Math. Sci., 2010, Article ID 369078, 15 pages. http://dx.doi.org/10.1155/2010/369078

27. Al-Refai, O., \& Darus, M. (2009). Main differential sandwich theorem with some applications. Lobachevskii J. Math., 30(1), 1-11. https://doi.org/10.1134/S1995080209010016

28. Ibrahim, R. W., \& Darus, M. (2010). Differential Subordination for classes of normalized analytic functions. General Mathematics, 18(3), 41-50.

29. Ibrahim, R. W., \& Darus, M. (2010). On Certain classes of multivalent analytic functions. Journal of Mathematics and Statistics, 6(3), 271-275. https://doi.org/10.3844/jmssp.2010.271.275

\section{Abdussalam Eghbiq}

School of Mathematical Sciences, Faculty of Science and Technology, Universiti Kebangsaan Malaysia, Selangor, DE, Bangi UKM 43600, Malaysia.

e-mail: eghbiq@gmail.com

Maslina Darus

School of Mathematical Sciences, Faculty of Science and Technology, Universiti Kebangsaan Malaysia, Selangor, DE, Bangi UKM 43600, Malaysia.

e-mail: maslina@ukm.edu.my 